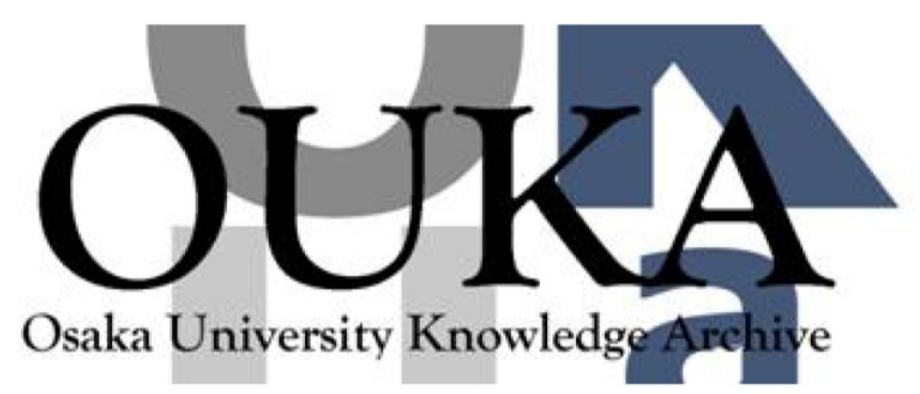

\begin{tabular}{|c|c|}
\hline Title & $\begin{array}{l}\text { Elastic-stiffness mapping by resonance- } \\
\text { ultrasound microscopy with isolated } \\
\text { piezoelectric oscillator }\end{array}$ \\
\hline Author(s) & $\begin{array}{l}\text { Ogi, Hirotsugu; Tian, Jiayong; Tada, Toyokazu } \\
\text { et al. }\end{array}$ \\
\hline Citation & Applied Physics Letters. 83(3) p.464-p. 466 \\
\hline Issue Date & $2003-07-21$ \\
\hline oaire:version & VoR \\
\hline URL & https://hdl. handle. net/11094/84203 \\
\hline rights & $\begin{array}{l}\text { This article may be downloaded for personal use } \\
\text { only. Any other use requires prior permission } \\
\text { of the author and AIP Publishing. This article } \\
\text { appeared in Applied Physics Letters, } 83(3), 464 \\
\text { (2003) and may be found at } \\
\text { https://doi.org/10.1063/1.1593819. }\end{array}$ \\
\hline Note & \\
\hline
\end{tabular}

Osaka University Knowledge Archive : OUKA

https://ir. Library. osaka-u. ac. jp/

Osaka University 


\section{Elastic-stiffness mapping by resonance- ultrasound microscopy with isolated piezoelectric oscillator}

Cite as: Appl. Phys. Lett. 83, 464 (2003); https://doi.org/10.1063/1.1593819

Submitted: 03 April 2003. Accepted: 02 June 2003 . Published Online: 16 July 2003

Hirotsugu Ogi, Jiayong Tian, Toyokazu Tada, and Masahiko Hirao

ARTICLES YOU MAY BE INTERESTED IN

Young's modulus mapping on SCS- $6 \mathrm{SiC}_{f} / \mathrm{Ti}-6 \mathrm{Al}-4 \mathrm{~V}$ composite by electromagnetic-resonanceultrasound microscopy

Journal of Applied Physics 94, 6472 (2003); https://doi.org/10.1063/1.1623611

Internal-friction mapping on solids by resonance ultrasound microscopy

Applied Physics Letters 88, 141110 (2006); https://doi.org/10.1063/1.2194479

Implementation of a modern resonant ultrasound spectroscopy system for the measurement of the elastic moduli of small solid specimens

Review of Scientific Instruments 76, 121301 (2005); https://doi.org/10.1063/1.2140494

\section{Challenge us.}

What are your needs for periodic signal detection?

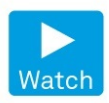

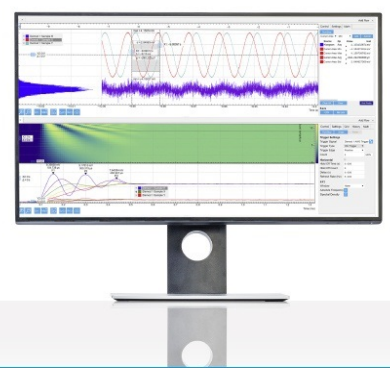

- Zurich - Instruments 


\title{
Elastic-stiffness mapping by resonance-ultrasound microscopy with isolated piezoelectric oscillator
}

\author{
Hirotsugu Ogi, ${ }^{\text {a) }}$ Jiayong Tian, Toyokazu Tada, and Masahiko Hirao \\ Graduate School of Engineering Science, Osaka University, Machikaneyama 1-3, Toyonaka, \\ Osaka 560-8531, Japan
}

(Received 3 April 2003; accepted 2 June 2003)

\begin{abstract}
A resonance-ultrasound microscopy has been developed for mapping a material's elastic constant in a localized surface region. It detects the effective elastic modulus through a resonance frequency of free vibrations of a solid probe touching the specimen via a small tungsten-carbide bearing. Langasite $\left(\mathrm{La}_{3} \mathrm{Ga}_{5} \mathrm{SiO}_{14}\right)$ crystal is used as a probe because of the low sensitivity of its elastic constants to temperature and its high piezoelectric coefficients. The vibration of the probe is excited and detected with a surrounding solenoid coil. This noncontacting acoustic coupling isolates the probe vibration and measures the resonance frequency with an accuracy better than one part in $10^{5}$. This microscopic method is applied to a composite material consisting of silicon-carbide ( $\mathrm{SiC}$ ) fibers in titanium-alloy matrix. The stiffness distribution inside a single fiber was determined.
\end{abstract} (C) 2003 American Institute of Physics. [DOI: 10.1063/1.1593819]

Microscale and nanoscale elastic-constant mapping has been recently made possible with atomic-force acoustic microscopy (AFAM) ${ }^{1-4}$ This method yields an image of the effective stiffness of a surface region of a material from the resonance frequency of an atomic-force-microscope (AFM) cantilever touching the material. The image includes information about the material's local elastic constants, which is unavailable with other conventional observations, such as optical microscopy, scanning and transmission electron microscopy, and AFM topography. However, with this approach, it is difficult to make an absolute quantitative determination of the material's stiffness due to many unstable and unknown parameters. The typical AFAM setup uses an ultrasonic transducer attached to the cantilever to vibrate it, causing a multiple resonance together with the transducer, bonding material between the transducer and the cantilever, and the cantilever. One end of the cantilever is gripped and the other end contacts the specimen. Thus, the resonance frequencies depend not only on the specimen's stiffness, but also highly on the elastic constants of the transducer material, coupling material, cantilever material, and gripping material. In addition, the gripping condition enters the problem. It highly affects the resonance frequencies because maximum bending and torsional stresses always occur at the gripped surface of the cantilever. To overcome these problems, we must isolate the probe vibration. Furthermore, the usual AFAM approach is an unrealistic means for nondestructive evaluation of materials in the field because it is too sensitive to the environmental conditions, such as temperature and sound noises, to provide a stable resonance frequency.

Here, we present a resonance-ultrasound microscopy using a piezoelectric crystal [langasite, $\mathrm{La}_{3} \mathrm{Ga}_{5} \mathrm{SiO}_{14}$ (LGS)], instead of a cantilever. LGS belongs to crystals with 32 point-group symmetry (trigonal) that show six independent elastic constants $C_{i j k l}$, two piezoelectric coefficients $e_{i j k}$,

${ }^{a)}$ Electronic mail: ogi@me.es.osaka-u.ac.jp and two dielectric coefficients $\varepsilon_{i j} .{ }^{5}$ Its large piezoelectric coefficients (about three times as large as those of quartz) allow us to oscillate the crystal without contact using a dynamic electric field. In addition, because its elastic constants are hardly affected by temperature (normalized temperature derivatives of $C_{i j k l}$ are of the order of $\left.10^{-5} \mathrm{~K}^{-1}\right),{ }^{5}$ the resonance frequencies are very stable.

Figure 1 illustrates the microscopy method. An oriented rectangular parallelepiped LGS probe is used, measuring about $10 \times 10 \times 14 \mathrm{~mm}^{3}$. The solenoid coil surrounds the probe without touching it. Driving the solenoid coil by highpower if bursts induces an oscillating electric field and excites free vibrations of the probe through the converse piezoelectric effect. After the excitation, the same coil receives the vibration through the piezoelectric effect. A frequency scan provides many resonance peaks as shown in Fig. 2. A tungsten-carbide spherical bearing $0.7 \mathrm{~mm}$ in diameter is embedded in a thin bearing base attached at an antinode spot of a vibration on the bottom surface of the probe. The bearing can rotate, contacting the specimen to measure the effective modulus in the near acoustic field. A biasing force is applied through three pins touching vibrational nodes on the top sur-

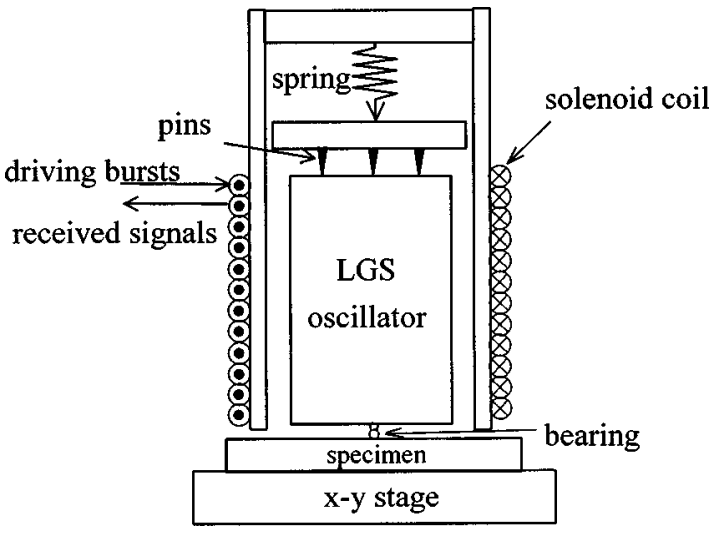

FIG. 1. Illustration of the rectangular-parallelepiped LGS oscillator, touching the specimen through a ball bearing attached at an antinode spot. 


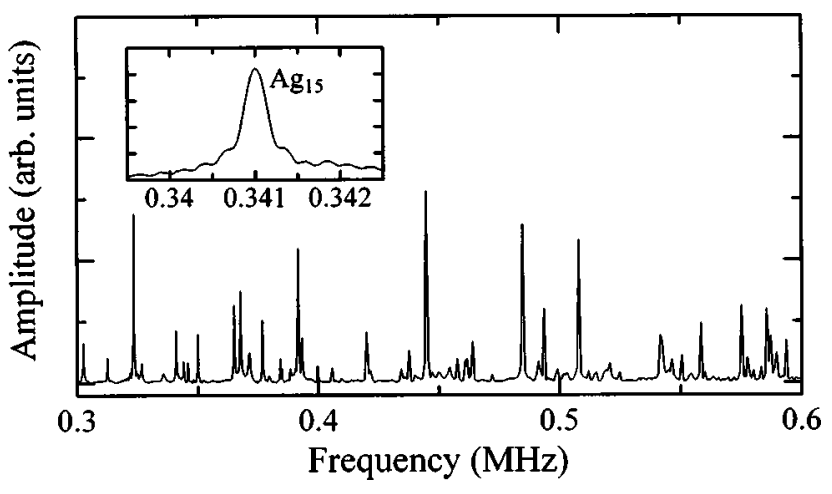

FIG. 2. Free-vibration resonance spectrum of the LGS crystal measured by the noncontacting solenoid coil.

face. Thus, an acoustic coupling occurs only at the specimen surface, and the vibration of the probe is isolated from any other contacts, realizing high sensitivity and reproducibility of the measurement.

Free vibrations of an oriented rectangular-parallelepiped crystal with 32 point-group symmetry fall into four groups denoted by $A_{g}, B_{g}, A_{u}$, and $B_{u}$, according to the deformation symmetry as tabulated by Ohno. ${ }^{6}$ Seeking the resonance frequencies and vibration modes, we consider the weak form of the Lagrangian in a piezoelectric material: ${ }^{6-9}$

$$
\begin{aligned}
L= & \frac{1}{2} \int_{V}\left(S_{i j} C_{i j k l} S_{k l}-\frac{\partial \phi}{\partial x_{m}} \varepsilon_{m n} \frac{\partial \phi}{\partial x_{n}}+2 \frac{\partial \phi}{\partial x_{m}} e_{m k l} S_{k l}\right. \\
& \left.-\rho \omega^{2} u_{i} u_{i}\right) d V-\int_{S} T_{k} u_{k} d S .
\end{aligned}
$$

Here, $S_{i j}$ denotes a component of the strain tensor, $\phi$ the electric potential, $\rho$ the mass density, and $\omega$ the angular frequency. $T_{k}$ and $u_{k}$ denote the surface traction and surface displacement, respectively, and they are related to the elastic properties of the contacting specimen. Assuming a point contact and using Hertz's theory, ${ }^{4}$ the out-of-plane component of the displacement at the contacting point $u_{3}^{\text {cont }}$ takes the form $u_{3}^{\text {cont }}=\left(F-F_{0}\right)\left(6 E^{* 2} R F_{0}\right)^{-1 / 3}$. Here $F_{0}$ denotes the biasing static force and $F$ the force acting on the probe in a resonance state. (We assumed $\left|F-F_{0}\right| \ll F_{0}$.) $F-F_{0}$ equals a perturbation force from the static force with a harmonic oscillation and can be integrated as a traction force acting at the contacting point in Eq. (1). $R$ is the bearing radius. $E^{*}$ denotes the effective Young's modulus of the bearing-specimen contact given by $E^{*-1}=E_{1}^{-1}\left(1-\nu_{1}^{2}\right)+E_{2}^{-1}\left(1-\nu_{2}^{2}\right)$. Here, $E$ and $\nu$ denote Young's modulus and Poisson's ratio, respectively. ${ }^{4}$ Subscripts 1 and 2 denote bearing and specimen, respectively. For the bearing, $E_{1}=630 \mathrm{GPa}$ and $\nu_{1}$ $=0.2$.

Stationary points of the Lagrangian $(\delta L=0)$ provide the resonance modes. We approximated the displacements and electric potential in terms of linear combinations of the basis functions consisting of normalized Legendre polynomials. ${ }^{6}$ Substituting the expanded displacements and electric potential into Eq. (1), we obtain the resonance frequencies together with the associated sets of expanding coefficients (Rayleigh-Ritz method). ${ }^{6-9}$ Resonance frequencies calculated using LGS constants reported previously ${ }^{5}$ agreed with the measurements by $0.1 \%$ difference in average when the
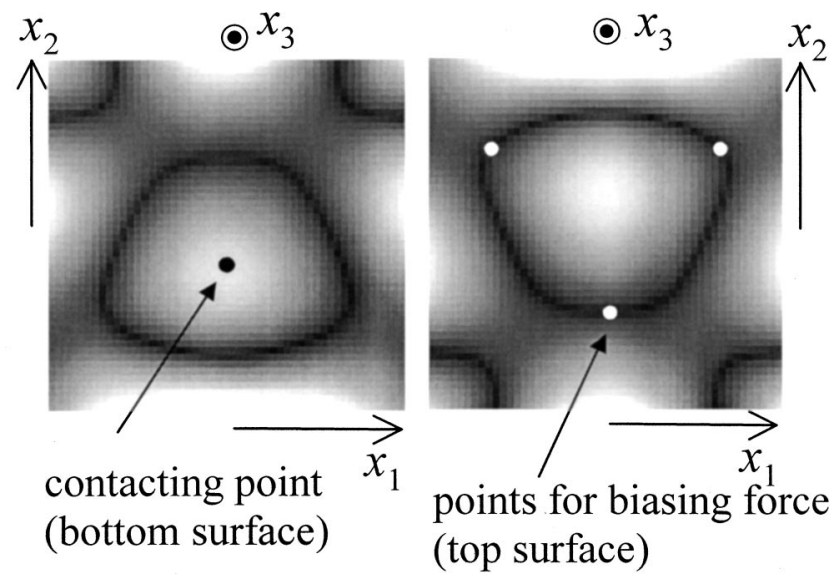

FIG. 3. Computed distributions of the out-of-plane displacement amplitude of $\mathrm{Ag}_{15}$ mode at the bottom (left) and top (right) surfaces of the LGS probe.

specimen is absent (free vibration). This agreement shows that the present method can determine a material's stiffness quantitatively. The expanding coefficients provide the displacement distributions on the probe surfaces as shown in Fig. 3. We also measured these using laser-Doppler interferometry and found good agreement with the computations, as demonstrated elsewhere. ${ }^{8,9}$

We used the $\mathrm{Ag}_{15}$ mode because it exhibits an antinode near the center of the bottom surface (Fig. 3). We fitted the spherical bearing on the center of the bottom surface (antinode) and applied the biasing force through the three pins touching the nodal points (Fig. 3). The temperature derivative of the resonance frequency of this mode was 2.3 $\times 10^{-5} \mathrm{~K}^{-1}$. Thus, the resonance frequency is stable because of both the isolation of the vibration and the low temperature derivative. Indeed, reproducibility of the resonance frequency measurement was on the order of $10^{-6}$ at ambient temperature.

Figure 4 shows the resonance frequency measured on various materials when $F_{0}=1.36 \mathrm{~N}$. Higher-stiffness specimens constrain the displacement $u_{3}^{\text {cont }}$ and increase the resonance frequency. (Detailed analysis of the contacting effect on the resonance frequency will be given elsewhere.) Figure

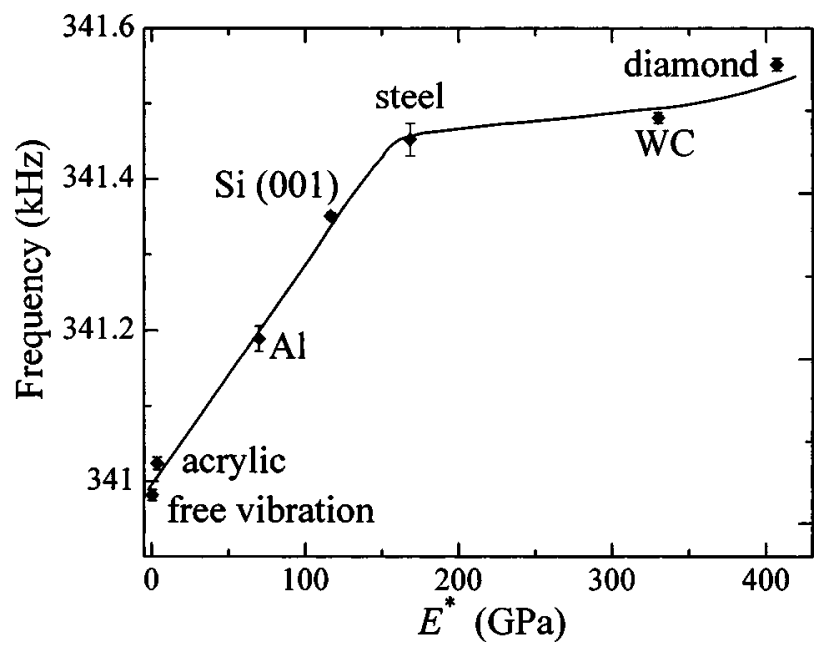

FIG. 4. Measured resonance frequency for six various materials with a wide range of elastic stiffnesses. 


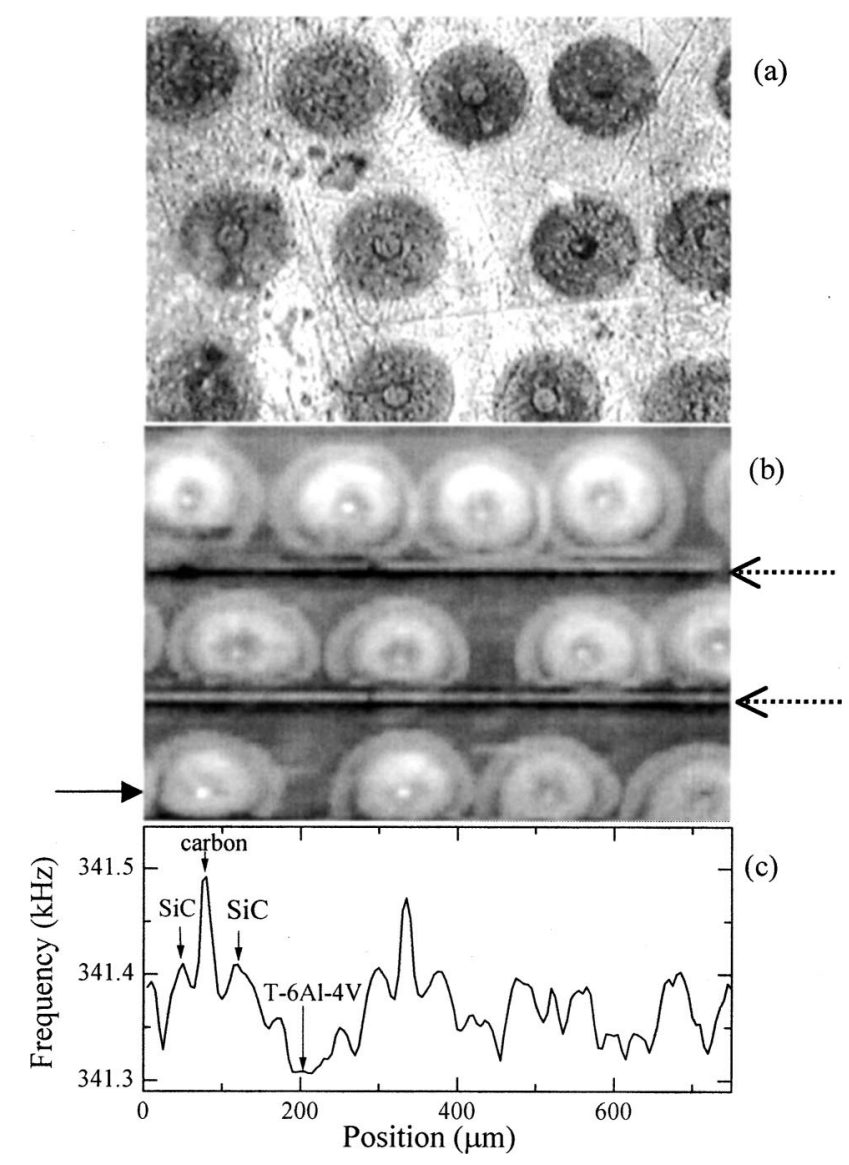

FIG. 5. Images obtained by (a) optical microscopy and (b) resonanceultrasound microscopy. (c) Line trace of the resonance frequency along the solid-line arrow shown in (b). Intensities differ because fibers are misaligned slightly. Dashed-line arrows show the interfaces of the matrix foils and fiber foils. (The foil layers are parallel to the horizontal direction.) The specimen surface was polished mechanically.

5 compares images obtained by optical microscopy and by resonance-ultrasound microscopy on a mechanically polished cross section of composite material consisting of a polycrystalline Ti-6Al-4V matrix reinforced unidirectionally by silicon-carbide (SiC) fibers (SCS-6). ${ }^{10}$ The single SCS-6 $\mathrm{SiC}$ fiber shows an annular structure consisting of a carbon core, $\mathrm{SiC}$ surrounding the core, and a carbon coating on the outer skin. ${ }^{11} \mathrm{~A}$ linear trace of the resonance frequency along the solid-line arrow in Fig. 5(b) is also shown in Fig. 5(c). The stiffness image reveals the effective Young's moduli of the carbon core and the matrix alloy larger and smaller than that of the $\mathrm{SiC}$, respectively. The calibration measurement in
Fig. 4 estimates Young's moduli of the matrix and carbon core to be 106 and $550 \mathrm{GPa}$, respectively. The former is consistent with that of the titanium alloy. The carbon core of SCS-6 SiC fiber includes a pyrolitic-carbon layer, which consists of turbostratic-carbon blocks with $c$-axes aligned preferentially along the radial direction. ${ }^{11}$ Thus, the larger modulus in the carbon core is attributed to the aligned pyroliticcarbon layer. There are two other significant observations, which are unavailable with optical microscopy. First, the modulus in the $\mathrm{SiC}$ region near the carbon core is small. It is reported that the concentration of the carbon atoms decreases with the radial distance from the edge of the carbon core and that it reaches the stoichiometric proportion in the $\mathrm{SiC}$ region at about $15 \mu \mathrm{m}$ from the edge. ${ }^{11}$ Thus, the $\mathrm{SiC}$ near the carbon core contains extra carbon atoms and shows a stiffness smaller than that of stoichiometric SiC. Second, the modulus at the interfaces between the matrix foils and fiber foils [dashed-line arrows in Fig. 5(b)] is small. Because this composite was fabricated by a foil-fiber-foil technique, ${ }^{10}$ the interfaces between the foils will be bonded poorly.

In summary, we developed a resonance-ultrasound microscopy for scanning elastic-stiffness changes across a planar specimen surface. Two factors enabled this method. First, we isolated the probe vibration. Second, we constructed a probe whose vibration is undisturbed by temperature change. As an example application, the microscopy method was capable of evaluating elastic-stiffness distribution across an $\mathrm{SiC}_{f} /$ titanium-matrix composite material. Not only did it show clearly the fiber/matrix elastic-constant changes, but it revealed further essential details. It would be easy to construct mobile probe for extending the method to field applications.

${ }^{1}$ K. Yamanaka, H. Ogiso, and O. Kolosov, Appl. Phys. Lett. 64, 178 (1994).

${ }^{2}$ O. Wright and N. Nishiguchi, Appl. Phys. Lett. 71, 626 (1997).

${ }^{3}$ U. Rabe, J. Turner, and W. Arnold, Appl. Phys. A: Mater. Sci. Process. 66, S277 (1998).

${ }^{4}$ G. G. Yaralioglu, F. L. Degertekin, K. B. Crozier, and C. F. Quate, J. Appl. Phys. 87, 7491 (2000).

${ }^{5}$ A. Bungo, C. Jian, K. Yamaguchi, Y. Sawada, S. Uda, and Y. Pisarevsky, Jpn. J. Appl. Phys. 38, 3239 (1999).

${ }^{6}$ I. Ohno, Phys. Chem. Miner. 17, 371 (1990).

${ }^{7}$ P. R. Heyliger, J. Acoust. Soc. Am. 107, 1235 (2000).

${ }^{8}$ H. Ogi, Y. Kawasaki, M. Hirao, and H. Ledbetter, J. Appl. Phys. 92, 2451 (2002).

${ }^{9}$ H. Ogi, N. Nakamura, K. Sato, M. Hirao, and S. Uda, IEEE Trans. Ultrason. Ferroelectr. Freq. Control 50, 553 (2003).

${ }^{10}$ J. Wadsworth and F. H. Froes, J. Met. 41, 12 (1989).

${ }^{11}$ X. J. Ning and P. Pirouz, J. Mater. Res. 6, 2234 (1991). 